\title{
A Plane-Symmetric Inhomogeneous Cosmological Model of Perfect Fluid Distribution with Electromagnetic Field I
}

\author{
Anirudh Pradhar1, Prashant Kumar Singh², Anil Kumar Yadav ${ }^{3}$ \\ ${ }^{1,2}$ Department of Mathematics, Hindu Post-graduate College, Zamania-232 \\ 331, Ghazipur, India \\ ${ }^{1}$ E-mail: pradhan@iucaa.ernet.in \\ ${ }^{3}$ Department of Physics, K. N. Govt. Post-graduate College, Sant Ravidas \\ Nagar (Gyanpur), Bhadohi-221 304, India \\ abanilyadav@yahoo.co.in
}

\begin{abstract}
A plane-symmetric inhomogeneous cosmological model of perfect fluid distribution with electro-magnetic field is obtained. The source of the magnetic field is due to an electric current produced along the z-axis. $F_{12}$ is the non-vanishing component of electromagnetic field tensor. To get a deterministic solution, we assume the free gravitational field is Petrov type-II non-degenerate. The behaviour of the electro-magnetic field tensor together with some physical aspects of the model are also discussed.
\end{abstract}

Key words : Cosmology, Electromagnetic field, Inhomogeneous solution . PACS: 98.80.Jk, 98.80.-k

\section{Introduction}

The standard Friedman-Robertson-Walker (FRW) cosmological model prescribes a homogeneous and an isotropic distribution for its matter in the description of the present state of the universe. At the present state of evolution, the universe is spherically symmetric and the matter distribution in the universe is on the whole isotropic and homogeneous. But in early stages of evolution, it could have not had such a smoothed picture. Close to the big bang singularity, neither the assumption of spherical symmetry nor that of isotropy can be strictly valid. So we consider plane-symmetric, which is less restrictive than spherical symmetry and can provide an avenue to study inhomogeneities. Inhomogeneous cosmological models play an important role in understanding some essential features of the universe such as the formation of galaxies during the early stages of evolution and process of homogenization. The early attempts at the construction of

${ }^{1}$ Corresponding author 
such models have done by Tolman [1] and Bondi [2] who considered spherically symmetric models. Inhomogeneous plane-symmetric models were considered by Taub [3, 4] and later by Tomimura [5], Szekeres [6], Collins and Szafron [7], Szafron and Collins [8]. Recently, Senovilla [9] obtained a new class of exact solutions of Einstein's equation without big bang singularity, representing a cylindrically symmetric, inhomogeneous cosmological model filled with perfect fluid which is smooth and regular everywhere satisfying energy and causality conditions. Later, Ruis and Senovilla [10] have separated out a fairly large class of singularity free models through a comprehensive study of general cylindrically symmetric metric with separable function of $r$ and $t$ as metric coefficients. Dadhich et al. 11] have established a link between the FRW model and the singularity free family by deducing the latter through a natural and simple inhomogenization and anisotropization of the former. Recently, Patel et al. [12] presented a general class of inhomogeneous cosmological models filled with nonthermalized perfect fluid by assuming that the background space-time admits two space-like commuting killing vectors and has separable metric coefficients. Bali and Tyagi [13, obtained a plane-symmetric inhomogeneous cosmological models of perfect fluid distribution with electro-magnetic field. Recently, Pradhan et al. 14] have investigated plane-symmetric inhomogeneous cosmological models in different context.

The occurrence of magnetic fields on galactic scale is well-established fact today, and their importance for a variety of astrophysical phenomena is generally acknowledged as pointed out by Zeldovich et al. [15. Also Harrison [16 has suggested that magnetic field could have a cosmological origin. As a natural consequences, we should include magnetic fields in the energy-momentum tensor of the early universe. The choice of anisotropic cosmological models in Einstein system of field equations leads to the cosmological models more general than Robertson-Walker model [17. The presence of primordial magnetic fields in the early stages of the evolution of the universe has been discussed by several authors [18 - 27. Strong magnetic fields can be created due to adiabatic compression in clusters of galaxies. Large-scale magnetic fields give rise to anisotropies in the universe. The anisotropic pressure created by the magnetic fields dominates the evolution of the shear anisotropy and it decays slower than if the pressure was isotropic 28, 29. Such fields can be generated at the end of an inflationary epoch 30-34. Anisotropic magnetic field models have significant contribution in the evolution of galaxies and stellar objects. Bali and Ali 35] had obtained a magnetized cylindrically symmetric universe with an electrically neutral perfect fluid as the source of matter. Pradhan et al. 36] have investigated magnetized viscous fluid cosmological models in different context.

Bali and Tyagi [37] have investigated a plane-symmetric inhomogeneous cosmological model of perfect fluid distribution with electromagnetic field. In this paper, we have revisited their solution and obtained a new plane-symmetric inhomogeneous cosmological model of perfect fluid distribution with electromagnetic field. To get deterministic solution, we consider the free gravitational field is Petrov type-II non-degenerate in which the distribution is that of perfect fluid. The paper is organized as follows. The metric and the field equations 
are presented in Section 2. In Section 3, we deal with the solution of the field equations. Section 4 includes the physical and geometric features of the models. Finally the results are discussed in Section 5 .

\section{The metric and field equations}

We consider the metric in the form

$$
d s^{2}=A^{2}\left(d x^{2}-d t^{2}\right)+B^{2} d y^{2}+C^{2} d z^{2},
$$

where the metric potential $A, B$ and $C$ are functions of $x$ and $t$. The energy momentum tensor is taken as

$$
T_{i}^{j}=(\rho+p) v_{i} v^{j}+p g_{i}^{j}+E_{i}^{j},
$$

where $E_{i}^{j}$ is the electro-magnetic field given by Lichnerowicz [38] as

$$
E_{i}^{j}=\bar{\mu}\left[h_{l} h^{l}\left(v_{i} v^{j}+\frac{1}{2} g_{i}^{j}\right)-h_{i} h^{j}\right] .
$$

Here $\rho$ and $p$ are the energy density and isotropic pressure respectively and $v^{i}$ is the flow vector satisfying the relation

$$
g_{i j} v^{i} v^{j}=-1
$$

$\bar{\mu}$ is the magnetic permeability and $h_{i}$ the magnetic flux vector defined by

$$
h_{i}=\frac{1}{\bar{\mu}}{ }^{*} F_{j i} v^{j}
$$

where ${ }^{*} F_{i j}$ is the dual electro-magnetic field tensor defined by Synge [39]

$$
{ }^{*} F_{i j}=\frac{\sqrt{-g}}{2} \epsilon_{i j k l} F^{k l} .
$$

$F_{i j}$ is the electro-magnetic field tensor and $\epsilon_{i j k l}$ is the Levi-Civita tensor density. The coordinates are considered to be comoving so that $v^{1}=0=v^{2}=v^{3}$ and $v^{4}=\frac{1}{A}$. We consider that the current is flowing along the z-axis so that $h_{3} \neq 0$, $h_{1}=0=h_{2}=h_{4}$. The only non-vanishing component of $F_{i j}$ is $F_{12}$. The Maxwell's equations

$$
F_{i j ; k}+F_{j k ; i}+F_{k i ; j}=0
$$

and

$$
\left[\frac{1}{\bar{\mu}} F^{i j}\right]_{; j}=J^{i}
$$

require that $F_{12}$ be function of $x$ alone. We assume that the magnetic permeability as a function of $x$ and $t$ both. Here the semicolon represents a covariant 
differentiation.

The Einstein's field equations ( in gravitational units $\mathrm{c}=1, \mathrm{G}=1$ ) read as

$$
R_{i}^{j}-\frac{1}{2} R g_{i}^{j}+\Lambda g_{i}^{j}=-8 \pi T_{i}^{j},
$$

for the line element (1) has been set up as

$$
\begin{gathered}
8 \pi A^{2}\left(p+\frac{F_{12}^{2}}{2 \bar{\mu} A^{2} B^{2}}\right)=-\frac{B_{44}}{B}-\frac{C_{44}}{C}+\frac{A_{4}}{A}\left(\frac{B_{4}}{B}+\frac{C_{4}}{C}\right) \\
+\frac{A_{1}}{A}\left(\frac{B_{1}}{B}+\frac{C_{1}}{C}\right)+\frac{B_{1} C_{1}}{B C}-\frac{B_{4} C_{4}}{B C}-\Lambda A^{2}, \\
8 \pi A^{2}\left(p+\frac{F_{12}^{2}}{2 \bar{\mu} A^{2} B^{2}}\right)=-\left(\frac{A_{4}}{A}\right)_{4}+\left(\frac{A_{1}}{A}\right)_{1}-\frac{C_{44}}{C}+\frac{C_{11}}{C}-\Lambda A^{2}, \\
8 \pi A^{2}\left(p-\frac{F_{12}^{2}}{2 \bar{\mu} A^{2} B^{2}}\right)=-\left(\frac{A_{4}}{A}\right)_{4}+\left(\frac{A_{1}}{A}\right)_{1}-\frac{B_{44}}{B}+\frac{B_{11}}{B}-\Lambda A^{2}, \\
8 \pi A^{2}\left(\rho+\frac{F_{12}^{2}}{2 \bar{\mu} A^{2} B^{2}}\right)=-\frac{B_{11}}{B}-\frac{C_{11}}{C}+\frac{A_{1}}{A}\left(\frac{B_{1}}{B}+\frac{C_{1}}{C}\right) \\
+\frac{A_{4}}{A}\left(\frac{B_{4}}{B}+\frac{C_{4}}{C}\right)-\frac{B_{1} C_{1}}{B C}+\frac{B_{4} C_{4}}{B C}+\Lambda A^{2}, \\
0=\frac{B_{14}}{B}+\frac{C_{14}}{C}-\frac{A_{1}}{A}\left(\frac{B_{4}}{B}+\frac{C_{4}}{C}\right)-\frac{A_{4}}{A}\left(\frac{B_{1}}{B}+\frac{C_{1}}{C}\right),
\end{gathered}
$$

where the sub indices 1 and 4 in $\mathrm{A}, \mathrm{B}, \mathrm{C}$ and elsewhere indicate ordinary differentiation with respect to $x$ and $t$, respectively.

\section{Solution of the field equations}

Equations (10) - (12) lead to

$$
\begin{gathered}
\left(\frac{A_{4}}{A}\right)_{4}-\frac{B_{44}}{B}+\frac{A_{4}}{A}\left(\frac{B_{4}}{B}+\frac{C_{4}}{C}\right)-\frac{B_{4} C_{4}}{B C}= \\
\left(\frac{A_{1}}{A}\right)_{1}+\frac{C_{11}}{C}-\frac{A_{1}}{A}\left(\frac{B_{1}}{B}+\frac{C_{1}}{C}\right)-\frac{B_{1} C_{1}}{B C}=\mathrm{a} \text { (constant) }
\end{gathered}
$$

and

$$
\frac{8 \pi F_{12}^{2}}{\bar{\mu} B^{2}}=\frac{B_{44}}{B}-\frac{B_{11}}{B}+\frac{C_{11}}{C}-\frac{C_{44}}{C} .
$$

Eqs. (10) - (14) represent a system of five equations in six unknowns $A, B, C, \rho$, $p$ and $\Lambda$. For the complete determination of these unknowns one more condition is needed. As in the case of general-relativistic cosmologies, the introduction 
of inhomogeneities into the cosmological equations produces a considerable increase in mathematical difficulty: non-linear partial differential equations must now be solved. In practice, this means that we must proceed either by means of approximations which render the non-linearities tractable, or we must introduce particular symmetries into the metric of the space-time in order to reduce the number of degrees of freedom which the inhomogeneities can exploit. In the present case, we assume that the metric is Petrov type-II non-degenerate. This requires that

$$
\begin{aligned}
& \left(\frac{B_{11}+B_{44}+2 B_{14}}{B}\right)-\left(\frac{C_{11}+C_{4}+2 C_{14}}{C}\right)= \\
& \frac{2\left(A_{1}+A_{4}\right)\left(B_{1}+B_{4}\right)}{A B}-\frac{2\left(A_{1}+A_{4}\right)\left(C_{1}+C_{4}\right)}{A C} .
\end{aligned}
$$

Let us consider that

$$
\begin{aligned}
& A=f(x) \lambda(t), \\
& B=g(x) \mu(t), \\
& C=g(x) \nu(t) .
\end{aligned}
$$

Using (18) in (14) and (17), we get

$$
\left[\frac{\frac{g_{4}}{g}-\frac{f_{1}}{f}}{\frac{g_{1}}{g}}\right]=\left[\frac{\frac{2 \lambda_{4}}{\lambda}}{\frac{\mu_{4}}{\mu}+\frac{\nu_{4}}{\nu}}\right]=\mathrm{b} \text { (constant) }
$$

and

$$
\frac{\frac{\mu_{44}}{\mu}-\frac{\nu_{44}}{\nu}}{\frac{\mu_{4}}{\mu}-\frac{\nu_{4}}{\nu}}-\frac{2 \lambda_{4}}{\lambda}=2\left(\frac{f_{1}}{f}-\frac{g_{1}}{g}\right)=\mathrm{L} \text { (constant). }
$$

Equation (19) leads to

$$
f=n g^{(1-b)}
$$

and

$$
\lambda=m(\mu \nu)^{\frac{b}{2}},
$$

where $\mathrm{m}$ and $\mathrm{n}$ are constants of integration. Equations (15), (18) and (20) lead to

$$
\left(\frac{b}{2}-1\right) \frac{\mu_{44}}{\mu}+(b-1) \frac{\mu_{4} \nu_{4}}{\mu \nu}=a
$$

and

$$
(2-b) \frac{g_{11}}{g}+(3 b-4) \frac{g_{1}^{2}}{g^{2}}=a .
$$

Let us assume

$$
\mu=e^{U+V}
$$

and

$$
\nu=e^{U-V}
$$


Equations (20), (25) and (26) lead to

$$
V_{4}=M e^{L t+2(b-1) U},
$$

where $\mathrm{M}$ is constant. From equations (23), (25), (26) and (27), we have

$$
(b-1) U_{44}+2(b-1) U_{4}^{2}-2 b M e^{L t+2(b-1) U} U_{4}-M L e^{L t+2(b-1) U}=a .
$$

If we put $e^{2 U}=\xi$ in equation (28), we obtain

$$
\frac{(b-1)}{2} \frac{d^{2} \xi}{d t^{2}}-M \frac{d}{d t}\left(e^{L t} \xi^{b}\right)=a \xi
$$

If we consider $\xi=e^{q t}$, then equation (29) leads to

$$
\frac{(b-1)}{2} g^{2} e^{q t}-M \frac{d}{d t}\left(e^{L t} e^{q b t}\right)=a e^{q t},
$$

which again reduces to

$$
q=\frac{L}{1-b}
$$

and

$$
a=\frac{L(L+2 M)}{2(b-1)} .
$$

Thus

$$
U=\frac{L t}{2(1-b)}
$$

Equations (27) and (33) reduce to

$$
V=M t+\log N,
$$

where $N$ is an integrating constant. Eq. (24) leads to

$$
g=\beta \cosh ^{\frac{2-b}{2(b-1)}}(\alpha x+\delta),
$$

where

$$
\alpha=\frac{\sqrt{2(3 b-4)(1-b)}}{(2-b)}
$$

and $\beta, \delta$ being constants of integration. Hence

$$
\begin{gathered}
f=n \beta \cosh ^{\frac{b-2}{2(b-1)}}(\alpha x+\delta), \\
\lambda=m e^{\frac{L t b}{2(1-b)}}, \\
\mu=e^{\frac{L t b}{2(1-b)}+M t+\log N}, \\
\nu=e^{\frac{L t b}{2(1-b)}-M t-\log N} .
\end{gathered}
$$


Therefore, we have

$$
\begin{gathered}
A=f \lambda=m n \beta e^{\frac{L t b}{2(1-b)}} \cosh ^{\frac{b-2}{2}}(\alpha x+\delta), \\
B=g \mu=N \beta e^{\left(\frac{L}{1-b}+2 M\right) \frac{t}{2}} \cosh ^{\frac{2-b}{2(b-1)}}(\alpha x+\delta), \\
C=g \nu=\frac{\beta}{N} e^{\left(\frac{L}{1-b}+2 M\right) \frac{t}{2}} \cosh ^{\frac{2-b}{2(b-1)}}(\alpha x+\delta) .
\end{gathered}
$$

By using the transformation

$$
\begin{gathered}
X=x+\frac{\delta}{\alpha}, \\
Y=y, \\
Z=z, \\
T=t,
\end{gathered}
$$

the metric (1) reduces to the form

$$
\begin{gathered}
d s^{2}=K^{2} \cosh ^{b-2}(\alpha X) e^{\frac{L T b}{1-b}}\left(d X^{2}-d T^{2}\right)+ \\
G^{2} \cosh ^{\frac{2-b}{b-1}}(\alpha X) e^{\left(\frac{L}{1-b}+2 M\right) T} d Y^{2}+H^{2} \cosh ^{\frac{2-b}{b-1}}(\alpha X) e^{\left(\frac{L}{1-b}-2 M\right) T} d Z^{2},
\end{gathered}
$$

where $K=m n \beta, G=N \beta$ and $H=\frac{\beta}{N}$.

\section{Some Physical and Geometric Features}

The physical parameters, pressure $(p)$ and density $(\rho)$, for the model (45) are given by

$$
\begin{gathered}
8 \pi p=\frac{1}{K^{2}} e^{\frac{m n b T}{b-1}} \cosh ^{2-b}(\alpha X)\left[\frac{(2-b)^{2} \alpha^{2}}{4(b-1)}\left\{1+\frac{2-b}{b-1} \tanh ^{2}(\alpha X)\right\}\right. \\
\left.-\frac{L^{2}}{4(1-b)^{2}}-M^{2}\right]-\Lambda, \\
8 \pi \rho=\frac{1}{K^{2}} e^{\frac{m n b T}{b-1}} \cosh ^{2-b}(\alpha X)\left[\frac{(2-b) \alpha^{2}}{2(b-1)}\left\{\frac{b}{b-1} \tanh ^{2}(\alpha X)-1\right\}\right. \\
\left.+\frac{L^{2}(2 b-1)}{4(1-b)^{2}}-M^{2}-\frac{M L}{(1-b)}\right]+\Lambda .
\end{gathered}
$$

The non-vanishing component $F_{12}$ of the electromagnetic field tensor is given by

$$
F_{12}=\sqrt{\frac{\bar{\mu}}{8 \pi} \frac{2 M L}{(1-b)}} G e^{\left(\frac{L}{1-b}+2 M\right) \frac{T}{2}} \cosh ^{\frac{2-b}{2(b-1)}}(\alpha X),
$$


where $\bar{\mu}$ remains undetermined as function of $x$ and $t$ both.

The scalar of expansion $(\theta)$ calculated for the flow vector $\left(v^{i}\right)$ is given by

$$
\theta=\frac{L(b+2)}{2 K(1-b)} e^{\frac{L b T}{2(b-1)}} \cosh ^{\frac{(2-b)}{2}}(\alpha X)
$$

The shear scalar $\left(\sigma^{2}\right)$, acceleration vector $\left(\dot{u}_{\mu}\right)$ and proper volume $\left(V^{3}\right)$ for the model (45) are given by

$$
\begin{gathered}
\sigma^{2}=\frac{\left(L^{2}+12 M^{2}\right)}{12 K^{2}} e^{\frac{L b T}{(b-1)}} \cosh ^{(2-b)}(\alpha X), \\
\dot{u}_{\mu}=\left(\frac{1}{2}(b-2) \alpha \tanh (\alpha X), 0,0,0\right), \\
V^{3}=K^{2} G H e^{\frac{L T(b+1)}{(1-b)}} \cosh ^{\frac{(b-2)(2 b+1)}{(b-1)}}(\alpha X) .
\end{gathered}
$$

From equations(49) and (50), we have

$$
\frac{\sigma^{2}}{\theta^{2}}=\frac{\left(L^{2}+12 M^{2}\right)\left(1-b^{2}\right)}{3 L^{2}(b+2)^{2}}=\text { constant }
$$

The rotation $\omega$ is identically zero and the non-vanishing component of conformal curvature tensor are given by

$$
\begin{gathered}
C_{(1212)}=\frac{1}{6 K^{2}} e^{\frac{L b T}{(b-1)}} \cosh ^{(2-b)}(\alpha X)\left[3 M L-2 M^{2}+b \alpha-\frac{L^{2}}{4 b}\right], \\
C_{(1313)}=\frac{1}{6 K^{2}} e^{\frac{L b T}{(b-1)}} \cosh ^{(2-b)}(\alpha X)\left[b \alpha-\frac{L^{2}}{4 b}-3 M L-2 M^{2}\right], \\
C_{(2323)}=\frac{1}{3 K^{2}} e^{\frac{L b T}{(b-1)}} \cosh ^{(2-b)}(\alpha X)\left[\frac{L^{2}}{4 b}-b \alpha+2 M^{2}+\frac{M L}{(1-b)}\right], \\
C_{(1224)}=\frac{M L}{2 K^{2}} e^{\frac{L b T}{(b-1)}} \cosh ^{(2-b)}(\alpha X) .
\end{gathered}
$$

The dominant energy condition is given by Hawking and Ellis 40]

$$
\begin{array}{cc}
\text { (i) } & \rho-p \geq 0 \\
\text { (ii) } & \rho+p \geq 0
\end{array}
$$

lead to

$$
\begin{gathered}
e^{\frac{m n b T}{(b-1)}}\left[\frac{(2-b) \alpha^{2}}{2(b-1)}\left\{\frac{\left(b^{2}-6 b+4\right)}{2(1-b)} \tanh ^{2}(\alpha X)+\frac{b-4}{2}\right\}\right. \\
\left.+\frac{L^{2} b}{2(1-b)^{2}}-\frac{M L}{(1-b)}\right]+2 K^{2} \Lambda \cosh ^{(b-2)}(\alpha X) \geq 0
\end{gathered}
$$

and

$$
\begin{gathered}
\frac{(2-b) \alpha^{2}}{4(b-1)^{2}}\left[\left(b^{2}-2 b+4\right) \tanh ^{2}(\alpha X)+b(1-b)\right] \geq \\
\frac{L^{2}}{2(1-b)}+2 M^{2}+\frac{M L}{(1-b)}
\end{gathered}
$$




\section{Conclusion}

We have obtained a new plane-symmetric inhomogeneous cosmological model of electro-magnetic perfect fluid as the source of matter. Generally the model represents expanding, shearing, non-rotating and Petrov type-II non-degenerate universe in which the flow vector is geodesic. We find that the model starts expanding at $T=0$ and goes on expanding indefinitely. However, if $b<0$ the process of contraction starts and at $T=\infty$ the expansion stops. For large values of $T$, the model is conformally flat and Petrov type-II non-degenerate otherwise. Since $\lim _{T \rightarrow \infty} \frac{\sigma}{\theta} \neq 0$. Therefore, the model does not approach isotropy for large values of $\mathrm{T}$. The electromagnetic field tensor does not vanish when $L \neq 0$, $M \neq 0$, and $b \neq 1$. For large values of t and $L+2 M(1-b)<0$ then $F_{12}$ tends to zero.

\section{Acknowledgements}

One of the authors (A. Pradhan) thanks Professor G. Date, IMSc., Chennai, India for providing facility where part of this work was carried out.

\section{References}

[1] R. C. Tolman, Proc. Nat. Acad. Sci. 20, 169 (1934).

[2] H. Bondi, Mon. Not. R. Astro. Soc. 107, 410 (1947).

[3] A. H. Taub, Ann. Math. 53, 472 (1951).

[4] A. H. Taub, Phy. Rev. 103, 454 (1956).

[5] N. Tomimura, II Nuovo Cimento B 44, 372 (1978).

[6] P. Szekeres, Commun. Math. Phys. 41, 55 (1975).

[7] C. B. Collins and D. A. Szafron, J. Math. Phy. 20, 2347 (1979a); J. Math. Phy. 20 (1979b) 2362.

[8] D. A. Szafron and C. B. Collins, J. Math. Phy. 20, 2354 (1979).

[9] J. M. M. Senovilla, Phy. Rev. Lett. 64, 2219 (1990).

[10] E. Ruiz and J. M. M. Senovilla, Phy. Rev. D 45, 1995 (1990).

[11] N. Dadhich, R. Tikekar and L. K. Patel, Curr. Sci. 65, 694 (1993).

[12] L. K. Patel, R. Tikekar and N. Dadhich, Pramana-J. Phys. 49, 213 (1993).

[13] R. Bali and A. Tyagi, Astrophys. Space Sc. 173, 233 (1990). 
[14] A. Pradhan, V. K. Yadav and N. N. Saste, Int. J. Mod. Phys. D 11, 857 (2002);

A. Pradhan and H. R. Pandey, Int. J. Mod. Phys. D 12, 941 (2003);

A. Pradhan and P. Pandey, Czech. J. Phys. 55, 749 (2005);

A. Pradhan, K. D. Thengane and J. K. Jumale, Spacetime \& Substance 4(29), 145 (2005);

A. Pradhan, P. Pandey and S. K. Singh, Int. J. Theor. Phys. (2007), to appear (gr-qc/0610125);

A. Pradhan, P. Pandey, K. Jotania and M. K. Yadav, Int. J. Theor. Phys. (2007), to appear.

[15] Ya. B. Zeldovich, A. A. Ruzmainkin and D. D. Sokoloff, Magnetic field in Astrophysics, (Gordon and Breach, New Yark, 1993).

[16] E. R. Horrison, Phys. Rev. Lett. 30, 188 (1973).

[17] H. P. Robertson and A. G. Walker, Proc. London Math. Soc. 42, 90 (1936).

[18] C. W. Misner, K. S. Thorne and J. A. Wheeler, Gravitation, (W. H. Freeman, New York, 1973).

[19] E. Asseo and H. Sol, Phys. Rep. 6, 148 (1987).

[20] M. A. Melvin, Ann. New York Acad. Sci. 262, 253 (1975).

[21] R. Pudritz and J. Silk, Astrophys. J. 342, 650 (1989).

[22] K. T. Kim, P. G. Tribble and P. P. Kronberg, Astrophys. J. 379, 80 (1991).

[23] R. Perley and G. Taylor, Astrophys. J. 101, 1623 (1991).

[24] P. P. Kronberg, J. J. Perry and E. L. Zukowski, Astrophys. J. 387, 528 (1991).

[25] A. M. Wolfe, K. Lanzetta and A. L. Oren: , Astrophys. J.388, 17 (1992).

[26] R. Kulsrud, R. Cen, J. P. Ostriker and D. Ryu, Astrophys. J. 380, 481 (1997).

[27] E. G. Zweibel and C. Heiles, Nature 385, 131 (1997).

[28] J. D. Barrow, Phys. Rev. D 55, 7451 (1997).

[29] Ya. A. Zeldovich, Sov. Astron. 13, 608 (1970).

[30] M. S. Turner and L. M. Widrow, Phys. Rev. D 30, 2743 (1988).

[31] J. Quashnock, A. Loeb and D. N. Spergel, Astrophys. J. 344, L49 (1989).

[32] B. Ratra, Astrophys. J. 391, L1 (1992). 
[33] A. D. Dolgov and J. Silk, Phys. Rev. D 47, 3144 (1993).

[34] A. D. Dolgov, Phys. Rev. D 48, 2499 (1993).

[35] R. Bali and M. Ali, Pramana 47, 25 (1996).

[36] I. Chakrabarty, A. Pradhan and N. N. Saste, Int. J. Mod. Phys. D 5, 741 (2001);

A. Pradhan and O. P. Pandey, Int. J. Mod. Phys. D 7, 1299 (2003);

A. Pradhan, S. K. Srivastav and K. R. Jotania, Czech. J. Phys. 54, 255 (2004);

A. Pradhan and S. K. Singh, Int. J. Mod. Phys. D 13, 503 (2004);

A. Pradhan, P. Pandey and K. K. Rai, Czech. J. Phys. 56, 303 (2006).

[37] R. Bali and A. Tyagi, Astrophys. Space Sci. 173, 233 (1990).

[38] A. Lichnerowicz, Relativistic Hydrodynamics and Magnetohydrodynamics, (W. A. Benzamin. Inc. New York, Amsterdam, p. 93 1967).

[39] J. L. Synge, Relativity: The General Theory, (North-Holland Publ., Amsterdam, p. 356, 1960).

[40] S. W. Hawking and G, F, R. Ellis, The Large-scale Structure of Space Time, (Cambridge University Press, Cambridge, p. 94, 1973). 